\title{
Energy-aware cluster based cooperative spectrum sensing for cognitive radio sensor networks
}

\begin{abstract}
Energy efficient spectrum sensing and data communication to extend the lifetime of cognitive radio sensor network is becoming increasingly important due to resource constraint of CRWSN inherent from WSN. This paper presents an energy-aware clustering (EAC) algorithm that enhances spectrum sensing performance and reduces network energy consumption thereby prolonging lifetime of the network. We derived network wide energy consumption model in terms of spectrum sensing energy consumption, intra cluster and inter clusters energy consumptions, and then determined the optimal number of clusters for the network. Through numerical analysis, we evaluate the effectiveness of the proposed algorithm in terms of minimizing network wide energy consumption and improving spectrum sensing performance.
\end{abstract}

Keyword: Cluster head; Clusters; Cognitive radio; Cooperative spectrum sensing; Energy consumption 